\title{
ChipSeg: An Automatic Tool to Segment Bacterial and Mammalian Cells Cultured in Microfluidic Devices
}

Irene de Cesare, Criseida G. Zamora-Chimal, Lorena Postiglione, Mahmoud Khazim, Elisa Pedone, Barbara Shannon, Gianfranco Fiore, Giansimone Perrino, Sara Napolitano, Diego di Bernardo, Nigel J. Savery, Claire Grierson, Mario di Bernardo, ${ }^{*}$, I and Lucia Marucci*, II

Cite This: ACS Omega 2021, 6, 2473-2476

Read Online

ABSTRACT: Extracting quantitative measurements from time-lapse images is necessary in external feedback control applications, where segmentation results are used to inform control algorithms. We describe ChipSeg, a computational tool that segments bacterial and mammalian cells cultured in microfluidic devices and imaged by time-lapse microscopy, which can be used also in the context of external feedback control. The method is based on thresholding and uses the same core functions for both cell types. It allows us to segment individual cells in high cell density microfluidic devices, to quantify fluorescent protein expression over a time-lapse experiment, and to track individual mammalian cells. ChipSeg enables robust segmentation in external feedback control experiments and can be easily customized for other experimental settings and research aims.

\section{INTRODUCTION}

Live-cell imaging by automated microscopy enables the collection of large-scale data useful to study the link between cellular dynamics and emerging phenotypes.

In the context of synthetic biology, the combination of control engineering algorithms with live-cell imaging has been shown to successfully enable the automatic regulation of gene expression across cellular chassis. ${ }^{1-8}$ If employing microfluidics/microscopy platforms, then the external feedback control action is implemented by measuring the relevant control output (e.g., fluorescent reporter expression in cells grown within microfluidic devices) throughout the time-lapse experiment by means of automatic segmentation. This measurement informs a control algorithm that computes the control input to minimize the control error (i.e., the difference between the control target and output). The control input is automatically provided to cells, for example, by changing the culture media with motor-controlled pumps. Automatic segmentation needs to be performed in real time and to be robust over the whole time-lapse experiment.

We present here ChipSeg, a thresholding-based algorithm that automatically segments both bacterial and mammalian cells cultured in microfluidic devices. The same core functions are implemented for both cell types, making the code flexible for other chassis and applications. The algorithm is easy to use and shows robust segmentation results in external feedback control experiments with microfluidics/microscopy platforms; ${ }^{5-10}$ ChipSeg can be easily adapted for open-loop experiments and other cell types/experimental settings.
ChipSeg is implemented in MATLAB, and it is publicly available. The source code and documentation can be found at https://github.com/LM-group/ChipSeg.

\section{RESULTS}

ChipSeg is broadly based on the Otsu thresholding method ${ }^{11}$ and is implemented in MATLAB using both custom and built-in Image Processing Toolbox functions. When used within an external feedback control experiment, the segmentation is performed online: at each sampling time, the acquired image is imported by ChipSeg and the algorithm output is fed back to the computer for control input calculation (Figure 1A,B).

First, ChipSeg performs global thresholding to segment a bacterial or mammalian cell cluster in a region of interest within the microfluidic device used for cell culturing; pre-processing, such as filtering and contrast enhancement, can be applied (Supporting Information). Global thresholding can be performed on phase-contrast images or, in the case of poor contrast, on images of cells stained with a fluorescent dye (Supporting Information).

Individual cells can also be identified using local thresholding for bacterial cells. The segmentation can be refined in a customized way to fix possible artifacts, for example, by

Received: August 13, 2020

Accepted: November 20, 2020

Published: January 19, 2021 

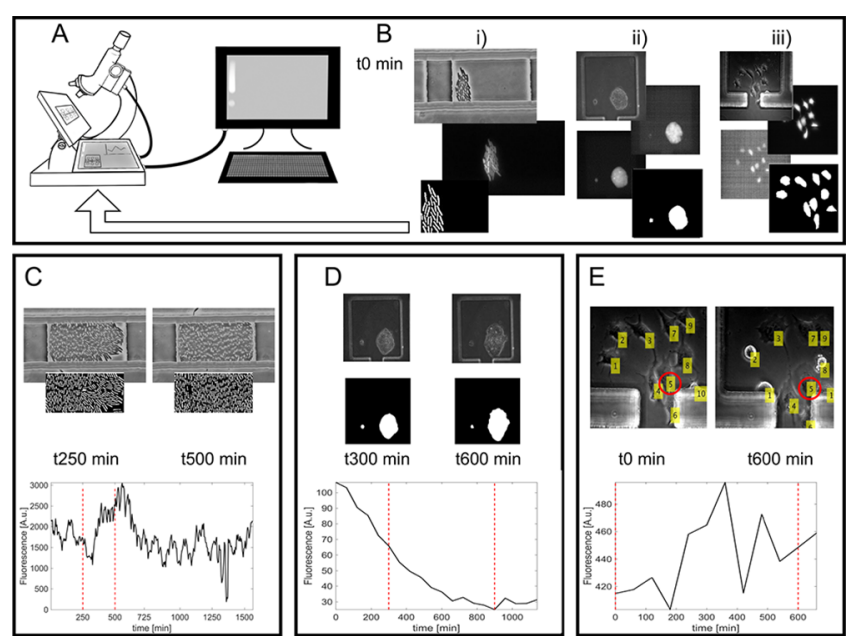

Figure 1. ChipSeg main features. (A, B) The algorithm can segment time-lapse images of bacterial (B-i) and mammalian cells with domeshaped (B-ii) or flat (B-ii) morphology, cultured in microfluidic devices; if used within an external feedback control experiment, then, at each sampling time, the image acquired by the microscope is segmented and the ChipSeg output is fed back to the computer, which can compute the control error and input. In panel (B), phase-contrast, fluorescence channels, and ChipSeg-computed masks (i, ii) and ellipsoids (iii) are shown. Masks are computed on a cropped area of the acquired raw image. (C, D) Average fluorescence quantification in exemplar bacterial cells (C) and Rex1-GFPd2 mouse embryonic stem cell (mESC; D) time lapses (Supporting Information); the dotted red lines indicate the time points for which the phase-contrast raw images and the corresponding ChipSeg-computed masks on a cropped region are shown. (E) Two time points (dotted red lines) of a dual-reporter mESC (Supporting Information) time lapse, showing the overlay of phase-contrast images and the ChipSeg-computed single-cell label tracked over time; quantification of the mCherry fluorescent reporter for a specific cell (labeled as 5) over the time lapse is shown.

removing or separating objects smaller/bigger than a set threshold, respectively (Supporting Information). A comparison with manually annotated images (i.e., ground truth) showed good segmentation results for both bacterial and mammalian cells (Supplementary Figures S8 and S15).

Fluorescence is calculated by overlapping the segmented masks to the fluorescence channel image; in doing so, a binary mask defining the pixels of the fluorescent image is calculated. Figure 1C,D and Supporting Movies S1 and S2 show the average fluorescence of cells within the selected region of interest for exemplar bacterial and mammalian cell time lapses, respectively.

If local thresholding is performed, then the algorithm provides the number of segmented cells, which can serve to estimate the cell population growth rate over the time-lapse experiment (Supporting Movie S1). Furthermore, by computing the maximum and minimum of fluorescence in the cellular population, cell-to-cell variability and fluoresce distribution can be quantified (Supporting Movie S1).

ChipSeg also allows for cell tracking, provided that the movement across frames is limited, and the microfluidic device is not too dense (in our examples, for flat mammalian cells). The algorithm first computes a mask by applying filters and thresholding functions, then assigns a centroid to an individual cell, and finally searches the correspondence between individual cells in two consecutive time-lapse images by minimizing the centroid displacement (Figure 1E and Supporting Movie S3).
Our algorithm is fully customizable as we provide a step-bystep explanation of all the pre-processing, segmentation, tracking, and fluorescence quantification functions (Supporting Information).

\section{DISCUSSION}

Many automated image segmentation tools exist to analyze microscopy images; ${ }^{12-14}$ user-friendly graphical interfaces within a software package are often presented as black-boxes which cannot be easily combined with other softwares (e.g., in external feedback control applications, where communication between segmentation and control code is required).

Other image analysis algorithms previously proposed to segment cells cultured and imaged within microfluidic devices are often specific for certain chip designs such as the mother machine device; ${ }^{15-19}$ instead, ChipSeg does not rely on the geometry of the devices we used (see refs 20 and 21 for bacterial and mammalian cell device description, respectively).

ChipSeg is based on simple MATLAB functions and should be easy to customize also for users lacking a computational background. While it is true that parameters within the algorithm might need to be tuned if using images of different cells or if varying the acquisition settings, a few trial-and-error iterations might be enough to fix them, even if using limited images. Segmentation of more complex images (e.g., tissues) might require different types of algorithms (e.g., refs 22 and 23).

Our algorithm has enough flexibility to be used across cell types, and adaptation of the code should be quicker than implementing deep learning-based approaches (e.g., refs 15-19 and 24-26), whose undeniable robustness, in most cases, comes at the cost of extensive algorithm training on manually annotated datasets.

\section{MATERIALS AND METHODS}

Bacterial Cell Microfluidic Device, Strain, and Image Acquisition. The microfluidic device used for the experiments shown here was developed by Mondragón-Palomino and colleagues; $^{21}$ soft lithography was used to make the device, as shown in ref 21 . Escherichia coli MG1655 cells carrying the comparator construct described in ref 27 were loaded in the device, as shown in ref 21 . For each experiment, time-lapse fluorescence microscopy experiments were performed using an inverted widefield fluorescence microscope (Leica) and images were taken using an Andor iXon Ultra digital camera. The microfluidic device was enclosed inside an incubation chamber set to $37^{\circ} \mathrm{C}$ (Pecon). The microscope took every 5 min images of cells growing inside the microfluidic device trapping chambers. At each time point, a phase contrast image (PhC) and a green fluorescent image were acquired for each of the three trapping chambers. Green fluorescent images were used for the detection of GFP. Images were acquired using a 100× objective. Exposure times were set to $100 \mathrm{~ms}$ for the $\mathrm{PhC}$ and green spectra.

Mammalian Cell Microfluidic Device, Cell Lines, and Image Acquisition. Experiments analyzed in this paper were performed with either of the two cell lines: a mouse embryonic stem cell (mESC) line stably carrying a destabilized GFP, replacing the entire Rex1 coding sequence in one allele (Rex1GFPd2), ${ }^{28}$ or dual-reporter mESCs carrying coding regions for eGFP and mCherry downstream of transcriptional start sites for mir-302 and mir-290 clusters, respectively. ${ }^{29}$ Cells were cultured on gelatinized tissue culture dishes at $37{ }^{\circ} \mathrm{C}$ in a $5 \% \mathrm{CO}_{2}$ 
humidified incubator in Dulbecco's modified Eagle medium (DMEM; Sigma; D5796) supplemented with 15\% fetal bovine serum (Sigma; F7524), nonessential amino acids (Gibco; 11140035), L-glutamine (Gibco; 25030024), sodium pyruvate (Gibco; 11360039), penicillin-streptomycin (Sigma; P4458), 2-mercaptoethanol (Gibco; 31350010), and $10 \mathrm{ng} / \mathrm{mL} \mathrm{mLIF}$ (Peprotech; 250-02). Both cell lines were transfected with a plasmid to express a transgene to encode nuclear Histone $2 \mathrm{~B}$ (H2B) tagged with infrared fluorescent protein (iRFP), as shown in ref 10 . The microfluidic device for mammalian cells was designed and characterized, as shown in ref 20. A master mold of the device (MicruX technologies) was used to produce device replicas by PDMS molding. Microfluidic device fabrication, processing, and cell loading into devices were performed, as previously described. ${ }^{20}$ Cells were vacuum-loaded into microfluidic chips and precultured with constant perfusion of fresh media while allowing to attach overnight in a humidified incubator; Rex1-GFPd2 cells were supplemented with $10 \mu \mathrm{M}$ cell permeable CellTracker blue (Invitrogen; C12881) and cultured in gelatine-treated chips; the microfluidic devices were precoated with fibronectin for dual-reporter $\mathrm{mESC}$ experiments. The device was secured onto a motorized microscope stage within an incubation chamber (incubator i8; Leica microsystems) and maintained at $37{ }^{\circ} \mathrm{C}$ in $5 \% \mathrm{CO}_{2}$. Time-lapse imaging was performed to acquire phase and fluorescent images using a Leica LASX live-cell imaging workstation on a DMi8 inverted fluorescence microscope with an Andor iXON 897 Ultra digital camera and a 40X objective (PlanFluor DLL 40× Ph2 Nikon). Adaptive focus control was employed on chosen fields, and images were acquired every $60 \mathrm{mins}$. The setup for the experiment in Figures S9-S11 (i.e., using the Rex1-GFPd2 $\mathrm{mESC}$ reporter line) consisted of four channels (phase contrast, green, blue, and infrared), while the setup for the experiment in Figures S12-S14 (i.e., using the dual reporter mESC line) consisted of three channels (phase contrast, red, and infrared).

\section{ASSOCIATED CONTENT}

\section{(s) Supporting Information}

The Supporting Information is available free of charge at https://pubs.acs.org/doi/10.1021/acsomega.0c03906.

Step-by-step explanation of the code (PDF).

Exemplar bacterial cell movie showing phase-contrast and GFP fluorescent images, ChipSeg-computed mask, average fluorescence, fluorescence distribution, and cell number (AVI).

Exemplar Rex1-GFPd2 mESC movie showing phasecontrast and green/blue fluorescent images as well as ChipSeg-computed mask and fluorescence (MP4).

Exemplar dual-reporter mESC movie showing phasecontrast and mCherry/infrared images, ChipSeg-computed cell labels and their tracking over time, and mCherry fluorescence of an individual cell (labeled as 5) over the time lapse (AVI).

\section{AUTHOR INFORMATION}

\section{Corresponding Authors}

Mario di Bernardo - Department of Engineering Mathematics and BrisSynBio, Life Sciences Building, University of Bristol, Bristol BS8 1UB, U.K.; Department of EE and ICT, University of Naples Federico II, 80125 Naples, Italy; ํ) orcid.org/00000002-2171-4745; Email: mario.dibernardo@unina.it
Lucia Marucci - Department of Engineering Mathematics, BrisSynBio, Life Sciences Building, and School of Cellular and Molecular Medicine, University of Bristol, Bristol BS8 1UB, U.K.; $\odot$ orcid.org/0000-0002-7553-6358;

Email: lucia.marucci@bristol.ac.uk

\section{Authors}

Irene de Cesare - Department of Engineering Mathematics, University of Bristol, Bristol BS8 1UB, U.K.

Criseida G. Zamora-Chimal - Department of Engineering Mathematics and BrisSynBio, Life Sciences Building, University of Bristol, Bristol BS8 1UB, U.K.

Lorena Postiglione - Department of Engineering Mathematics, University of Bristol, Bristol BS8 1UB, U.K.

Mahmoud Khazim - Department of Engineering Mathematics and School of Cellular and Molecular Medicine, University of Bristol, Bristol BS8 1UB, U.K.

Elisa Pedone - Department of Engineering Mathematics and School of Cellular and Molecular Medicine, University of Bristol, Bristol BS8 1UB, U.K.

Barbara Shannon - BrisSynBio, Life Sciences Building and School of Biochemistry, University of Bristol, Bristol BS8 1TQ, U.K.; $\odot$ orcid.org/0000-0003-2219-5462

Gianfranco Fiore - Department of Engineering Mathematics and BrisSynBio, Life Sciences Building, University of Bristol, Bristol BS8 1UB, U.K.; ○ orcid.org/0000-0002-5713-5323

Giansimone Perrino - Telethon Institute of Genetic and Medicine Via Campi Flegrei 34, 80078 Pozzuoli, Italy

Sara Napolitano - Telethon Institute of Genetic and Medicine Via Campi Flegrei 34, 80078 Pozzuoli, Italy

Diego di Bernardo - Telethon Institute of Genetic and Medicine Via Campi Flegrei 34, 80078 Pozzuoli, Italy; Department of Chemical, Materials and Industrial Production Engineering, University of Naples Federico II, 80125 Naples, Italy; (1) orcid.org/0000-0002-1911-7407

Nigel J. Savery - BrisSynBio, Life Sciences Building and School of Biochemistry, University of Bristol, Bristol BS8 1TQ U.K.; (1) orcid.org/0000-0002-0803-4075

Claire Grierson - BrisSynBio, Life Sciences Building and School of Biological Sciences, University of Bristol, Bristol BS8 1TQ, U.K.; o orcid.org/0000-0002-4000-0975

Complete contact information is available at:

https://pubs.acs.org/10.1021/acsomega.0c03906

\section{Author Contributions}

${ }^{\text {II } M . d . B . ~ a n d ~ L . M . ~ c o n t r i b u t e d ~ e q u a l l y ~ t o ~ t h i s ~ w o r k ~ a n d ~ s h o u l d ~ b e ~}$ considered co-senior authors.

\section{Author Contributions}

I.d.C. and C.G.Z.-C. contributed equally to this work and should be considered co-first authors.

\section{Author Contributions}

L.M. and M.d.B. conceived the project and supervised the work; L.P., G.F., G.P., and S.N. developed the initial code; I.d.C. and C.G.Z.-C. refined the algorithm and analyzed time-lapse experiments; M.K., B.S., and E.P. performed the experiments; D.d.B. supervised S.N. and G.P.; N.J.S. and C.G. co-supervised the experimental activities in bacteria; and I.d.C., C.G.Z.-C., and L.M. wrote the paper with input from the other authors.

\section{Funding}

This work was supported by BrisSynBio, a BBSRC/EPSRC Synthetic Biology Research Centre grant BB/L01386X/1 (to M.d.B. and L.M.), the EU Horizon 2020 research project COSY- 
BIO grant 766840 (to D.d.B., M.d.B., and L.M.), EPSRC grants EP/R041695/1 and EP/S01876X/1 (to L.M.), and MRC grant MR/N021444/1 (to L.M.).

\section{Notes}

The authors declare no competing financial interest.

\section{ACKNOWLEDGMENTS}

We thank Dr. Mark Jepson and Alan Leard (Wolfson Imaging Facility, University of Bristol) for supporting live-cell imaging experiments.

\section{REFERENCES}

(1) Milias-Argeitis, A.; Rullan, M.; Aoki, S. K.; Buchmann, P.; Khammash, M. Automated optogenetic feedback control for precise and robust regulation of gene expression and cell growth. Nat. Commun. 2016, 7, 12546.

(2) Lugagne, J.-B.; Sosa Carrillo, S.; Kirch, M.; Köhler, A.; Batt, G.; Hersen, P. Balancing a genetic toggle switch by real-time feedback control and periodic forcing. Nat. Commun. 2017, 8, 1671.

(3) Menolascina, F.; Fiore, G.; Orabona, E.; De Stefano, L.; Ferry, M.; Hasty, J.; di Bernardo, M.; di Bernardo, D. In-vivo real-time control of protein expression from endogenous and synthetic gene networks. PLoS Comput. Biol. 2014, 10, No. e1003625.

(4) Uhlendorf, J.; Miermont, A.; Delaveau, T.; Charvin, G.; Fages, F.; Bottani, S.; Batt, G.; Hersen, P. Long-term model predictive control of gene expression at the population and single-cell levels. Proc. Natl. Acad. Sci. U. S. A. 2012, 109, 14271-14276.

(5) Perrino, G.; Wilson, C.; Santorelli, M.; di Bernardo, D. Quantitative Characterization of $\alpha$-Synuclein Aggregation in Living Cells through Automated Microfluidics Feedback Control. Cell Rep. 2019, 27, 916-927.

(6) Postiglione, L.; Napolitano, S.; Pedone, E.; Rocca, D. L.; Aulicino, F.; Santorelli, M.; Tumaini, B.; Marucci, L.; di Bernardo, D. Regulation of Gene Expression and Signaling Pathway Activity in Mammalian Cells by Automated Microfluidics Feedback Control. ACS Synth. Biol. 2018, 7, 2558-2565.

(7) Pedone, E.; Postiglione, L.; Aulicino, F.; Rocca, D. L.; MontesOlivas, S.; Khazim, M.; di Bernardo, D.; Pia Cosma, M.; Marucci, L. A tunable dual-input system for on-demand dynamic gene expression regulation. Nat. Commun. 2019, 10, 4481.

(8) Fracassi, C.; Postiglione, L.; Fiore, G.; di Bernardo, D. Automatic Control of Gene Expression in Mammalian Cells. ACS Synth. Biol. 2016, 5, 296-302.

(9) Shannon, B.; Zamora-Chimal, C. G.; Postiglione, L.; Salzano, D.; Grierson, C. S.; Marucci, L.; Savery, N. J.; di Bernardo, M. In Vivo Feedback Control of an Antithetic Molecular-Titration Motif in Escherichia coli Using Microfluidics. ACS Synth. Biol. 2020, 9, 26172624.

(10) Khazim, M.; Postiglione, L.; Pedone, E.; Rocca, D. L.; Zahra, C.; Marucci, L. Towards automated control of embryonic stem cell pluripotency. IFAC-PapersOnLine 2019, 52, 82.

(11) Otsu, N. A Threshold Selection Method from Gray-Level Histograms. IEEE Trans. Syst. Man. Cybernetics 1979, 9, 62-66.

(12) Eliceiri, K. W.; Berthold, M. R.; Goldberg, I. G.; Ibañez, L.; Manjunath, B. S.; Martone, M. E.; Murphy, R. F.; Peng, H.; Plant, A. L.; Roysam, B.; Stuurman, N.; Swedlow, J. R.; Tomancak, P.; Carpenter, A. E. Biological imaging software tools. Nat. Methods 2012, 9, 697-710.

(13) Young, J. W.; Locke, J. C.; Altinok, A.; Rosenfeld, N.; Bacarian, T.; Swain, P. S.; Mjolsness, E.; Elowitz, M. B. Measuring single-cell gene expression dynamics in bacteria using fluorescence time-lapse microscopy. Nat. Protoc. 2011, 7, 80-88.

(14) Bajcsy, P.; Cardone, A.; Chalfoun, J.; Halter, M.; Juba, D.; Kociolek, M.; Majurski, M.; Peskin, A.; Simon, C.; Simon, M.; Vandecreme, A.; Brady, M. Survey statistics of automated segmentations applied to optical imaging of mammalian cells. BMC Bioinformatics 2015, 16, 330.
(15) Kaiser, M.; Jug, F.; Julou, T.; Deshpande, S.; Pfohl, T.; Silander, O. K.; Myers, G.; van Nimwegen, E. Monitoring single-cell gene regulation under dynamically controllable conditions with integrated microfluidics and software. Nat. Commun. 2018, 9, 212.

(16) Lugagne, J. B.; Lin, H.; Dunlop, M. J. DeLTA: Automated cell segmentation, tracking, and lineage reconstruction using deep learning. PLoS Comput. Biol. 2020, 16, No. el 1007673.

(17) Ollion, J.; Elez, M.; Robert, L. High-throughput detection and tracking of cells and intracellular spots in mother machine experiments. Nat. Protoc. 2019, 14, 3144-3161.

(18) Sachs, C. C.; Grünberger, A.; Helfrich, S.; Probst, C.; Wiechert, W.; Kohlheyer, D.; Noh, K. Image-Based Single Cell Profiling: HighThroughput Processing of Mother Machine Experiments. PLoS One 2016, 11, No. e0163453.

(19) Smith, A.; Metz, J.; Pagliara, S. MMHelper: An automated framework for the analysis of microscopy images acquired with the mother machine. Sci. Rep. 2019, 9, 10123.

(20) Kolnik, M.; Tsimring, L. S.; Hasty, J. Vacuum-assisted cell loading enables shear-free mammalian microfluidic culture. Lab Chip 2012, 12, 4732-4737.

(21) Mondragon-Palomino, O.; Danino, T.; Selimkhanov, J.; Tsimring, L.; Hasty, J. Entrainment of a population of synthetic genetic oscillators. Science 2011, 333, 1315-1319.

(22) Feng, Y.; Dong, F.; Xia, X.; Hu, C. H.; Fan, Q.; Hu, Y.; Gao, M.; Mutic, S. An adaptive Fuzzy $\mathrm{C}$-means method utilizing neighboring information for breast tumor segmentation in ultrasound images. Med Phys 2017, 44, 3752-3760.

(23) Feng, Y.; Kawrakow, I.; Olsen, J.; Parikh, P. J.; Noel, C.; Wooten, O.; Du, D.; Mutic, S.; Hu, Y. A comparative study of automatic image segmentation algorithms for target tracking in MR-IGRT. J Appl Clin Med Phys 2016, 17, 441-460.

(24) Stylianidou, S.; Brennan, C.; Nissen, S. B.; Kuwada, N. J.; Wiggins, P. A. SuperSegger: robust image segmentation, analysis and lineage tracking of bacterial cells. Mol. Microbiol. 2016, 102, 690-700.

(25) Falk, T.; Mai, D.; Bensch, R.; Cicek, O.; Abdulkadir, A.; Marrakchi, Y.; Bohm, A.; Deubner, J.; Jackel, Z.; Seiwald, K.; Dovzhenko, A.; Tietz, O.; Dal Bosco, C.; Walsh, S.; Saltukoglu, D.; Tay, T. L.; Prinz, M.; Palme, K.; Simons, M.; Diester, I.; Brox, T.; Ronneberger, O. U-Net: deep learning for cell counting, detection, and morphometry. Nat. Methods 2019, 16, 67-70.

(26) Van Valen, D. A.; Kudo, T.; Lane, K. M.; Macklin, D. N.; Quach, N. T.; DeFelice, M. M.; Maayan, I.; Tanouchi, Y.; Ashley, E. A.; Covert, M. W. Deep Learning Automates the Quantitative Analysis of Individual Cells in Live-Cell Imaging Experiments. PLoS Comput. Biol. 2016, 12, No. e1005177.

(27) Annunziata, F.; Matyjaszkiewicz, A.; Fiore, G.; Grierson, C. S.; Marucci, L.; di Bernardo, M.; Savery, N. J. An Orthogonal Multi-input Integration System to Control Gene Expression in Escherichia coli. ACS Synth. Biol. 2017, 6, 1816-1824.

(28) Wray, J.; Kalkan, T.; Gomez-Lopez, S.; Eckardt, D.; Cook, A.; Kemler, R.; Smith, A. Inhibition of glycogen synthase kinase- 3 alleviates Tcf3 repression of the pluripotency network and increases embryonic stem cell resistance to differentiation. Nat. Cell Biol. 2011, 13, 838-845.

(29) Parchem, R. J.; Ye, J.; Judson, R. L.; LaRussa, M. F.; Krishnakumar, R.; Blelloch, A.; Oldham, M. C.; Blelloch, R. Two miRNA clusters reveal alternative paths in late-stage reprogramming. Cell Stem Cell 2014, 14, 617-631. 